\title{
Combining Ability Studies in Pumpkin (Cucurbita moschata Duch ex Poir)
}

\author{
P. Marxmathi ${ }^{*}$, V. Krishnamoorthy ${ }^{1}$ and P. Thankaraj ${ }^{2}$ \\ ${ }^{1}$ Department of Horticulture, ${ }^{2}$ Department of Plant Breeding and Genetics, Agriculture \\ College and Research institute, Tamil Nadu Agricultural University, \\ Madurai- 625 104, Tamil Nadu, India \\ *Corresponding author
}

Keywords

Pumpkin, Cucurbita moschata, Sex ratio, Carotene, Yield

Article Info

Accepted:

26 February 2018

Available Online:

10 March 2018

\section{A B S T R A C T}

An experiment was conducted to study the combining ability analysis using six parental genotypes and thirty hybrids of pumpkin obtained through full diallel analysis for yield and its attributing traits. The mean sum of square due to general combining ability ( $\mathrm{gca}$ ) and specific combining ability ( $s c a$ ) were significant for nodes to first female flower, days to harvest, fruit weight, fruit length, fruit diameter and number of seeds per fruit. The ratio of $\mathrm{gca} / \mathrm{sca}$ was less than one for the character nodes to first female flower indicated the preponderance of dominance expression. The $\mathrm{P}_{1}$ is best combiner for days to first female flower (1.11) fruit weight (2.40) fruit length, fruit diameter and yield per plant. The $\mathrm{P}_{3}$ for more number fruits per plant (0.16), $\mathrm{P}_{4}$ for narrow sex ratio (2.06). $\mathrm{P}_{2}$ is best combiner for small sized fruits (0.21) which exhibited negative gca effects. The hybrids $\mathrm{P}_{1} \times \mathrm{P}_{2}(2.92)$, $\mathrm{P}_{1} \times \mathrm{P}_{2}(-0.44)$ and $\mathrm{P}_{4} \times \mathrm{P}_{1}(1.55)$ exhibited higher $s c a$ effect for yield and yield attributing traits. The highest $s c a$ effects were observed in $\mathrm{P}_{1} \times \mathrm{P}_{6}$ for node to first female flower (2.02), small fruit (-1.39), fruit diameter (1.86), in $\mathrm{P}_{2} \times \mathrm{P}_{1}$ for days to first female flower (2.94), bigger fruit weight (3.93), flesh thickness (0.24) and yield per plant (3.05), $\mathrm{P}_{4} \times \mathrm{P}_{1}$ for narrow sex ratio (-3.14) and carotene content in $\mathrm{P}_{5} \times \mathrm{P}_{4}$ for days to first female flower $(-1.63)$, flesh thickness (0.28), yield per plant (2.92) and beta carotene $(0.07)$.

\section{Introduction}

Pumpkin is one of important cucurbitaceous vegetable. It is sweet in nature and has very high content beta carotene which is cheapest source for all the people for the health of eyes. Three to four decades earlier most of our population with joint family in which the bigger sized fruits used by them. Now days the nucleus families increased in our population, the large sized fruits are not preferred and sometimes purchased as cut pieces. Many of the economic traits of genotypes are not associated with discrete Mendalian traits but they are quantitative nature. The yield of genotypes is also affected by the environmental factors. The basic information on combining ability in pumpkin is facilitates to select suitable parents for hybridization programme to develop promising $F_{1}$ hybrids and or hybrid derivatives. The concept of combining ability in terms of genetic variation was first given by Sprague and Tatum (1942) using single 
crosses in maize. The diallel cross help in determining general combining ability of parents and specific combining abilities of hybrids. Further it helps in formulating the breeding methodology for crop improvement. Hence, the present study was conducted with new set of diverse parental combinations to identify better parent and efficient hybrids of pumpkin for small fruit size, high yield and good quality through combining ability analysis.

\section{Materials and Methods}

The experiment was conducted with six parental genotypes viz., P1 (Acc.No. MDU CM23, Thirumangalam local, Madurai district) is high flesh thickness and medium sized fruit, P2 (Acc.No.MDU CM28, Oddanchatram local, Dinddugul district) is small fruited and more number of fruits, P3 (Acc.No. MDU CM29, Harur local, Dharmapuri distict) is early days to flowering and small fruited, P4 (Acc.No. MDU CM12, Department of Horticulture, AC \& RI Madurai) is high yield per plant, P5 (Acc.No. MDU CM1, Attur local, Salem district) is more flesh thickness, P6 (Acc.No. MDU CM31, Rajapalayam local, Virudhunagar district) is narrow sex ratio with medium sized fruits were selected from the germplam collection maintained at Dept. of Horticulture, AC\&RI, TNAU, Madurai. Six parental lines were crossed in all possible combinations including reciprocals to produce $F_{1}$ seeds by hand pollination using the method diallel cross (Griffing, 1956).

During December, 2016 all the seeds of 30 cross combinations and six parents were sown in randomized block design with two replications. Five plants were selected and tagged for recording the observations on different characters viz., vine length, number of primary branches, days to first male flower, days to first female flower, nodes to first male flower, nodes to first female flower, sex ratio, days to first harvest, number of fruits per vine, average fruit weight, fruit length, fruit diameter, flesh thickness, number of seeds per fruit, fruit yield per plant. All the cultural operations and plant protection measures were carried out as per the recommendations of crop production guide of Tamil Agricultural University, Coimbatore. The estimation of general and specific combining ability was done as per the procedures outlined by Griffing (1956) for method I of diallel analysis which included parents, $F_{1}$ and reciprocals using the following general formula for the model.

\section{Results and Discussion}

In the present study, mean squares (Table 1) due to gca were significant for vine length, days to first male flowering, days to first female flowering, nodes to first female flower, sex ratio, days to first harvest, average fruit weight, fruit length, fruit diameter, no. of seeds per fruit, fruit yield per plant, total soluble solid and moisture content in fruit. The mean squares due to $s c a$ were significant for nodes to first female flower, days to first harvest, average fruit weight, fruit length, fruit diameter and number of seeds per fruit. Mean squares in reciprocal were significant for vine length, days to first female flowering, nodes to female flower, sex ratio, days to first harvest, average fruit weight, fruit length, fruit diameter, number of seeds per fruit, and fruit yield per plant indicated the variation in parents and crosses and thus significant combination of additive and non-additive gene effects in the expression of the characters. The reciprocal variation might be due to cytoplasmic inheritance and its interaction with nuclear genes. Similar significant reciprocal effects reported earlier are bitter gourd by Gopalakrishanan (1986), Devadass (1993) and Thangamani and Pugalendhi (2013). 
Table.1 Analysis of variance for combining ability

\begin{tabular}{|c|c|c|c|c|c|}
\hline \multirow[t]{2}{*}{ Character } & \multicolumn{3}{|c|}{ Mean sum of square } & \multirow[b]{2}{*}{ Error } & \multirow[b]{2}{*}{$g c a / s c a$} \\
\hline & gca & sca & Reciprocal & & \\
\hline Vine length & $10.50^{* *}$ & 1.19 & $4.96 * *$ & 6.17 & 0.07 \\
\hline $\begin{array}{l}\text { No. of primary branches per } \\
\text { plant }\end{array}$ & 0.23 & 1.69 & 0.17 & 0.6 & 0.02 \\
\hline Days to first male flowering & $6.43 * *$ & 0.54 & 1.24 & 0.54 & 96.49 \\
\hline Days to first female flowering & $4.41 * *$ & 1.41 & $4.32 * *$ & 0.36 & 0.32 \\
\hline First male flower node & 1.56 & 0.83 & 0.61 & 0.25 & 0.18 \\
\hline First female flower node & $2.84 * *$ & $2.78^{* *}$ & $2.77 * *$ & 1.32 & 0.08 \\
\hline Sex ratio & $19.54^{* *}$ & 1.41 & $7.33 * *$ & 0.34 & 1.49 \\
\hline Days to fruit maturity & $12.39^{* *}$ & $2.02 *$ & $6.41 * *$ & 1.18 & 1.1 \\
\hline No of fruits per plant & 0.41 & 0.06 & 0.05 & 0.01 & 0.68 \\
\hline Fruit weight (kg) & $21.81 * *$ & $2.78 * *$ & $3.96 * *$ & 0.93 & -6.76 \\
\hline Fruit length $(\mathrm{cm})$ & $103.52 * *$ & $4.15^{* *}$ & $25.10^{* *}$ & 0.26 & 2.21 \\
\hline Fruit diameter $(\mathbf{c m})$ & $202.91 * *$ & $2.43 *$ & $69.04 * *$ & 0.98 & 11.64 \\
\hline Flesh thickness $(\mathrm{cm})$ & 0.28 & 0.049 & 0.14 & 0.01 & 0.64 \\
\hline No.of seeds/fruit & $2950.58 * *$ & $182.53 * *$ & $1715.59 * *$ & 26.97 & 1.56 \\
\hline Yield per plant & $23.11 * *$ & 1.74 & $8.41^{* *}$ & 0.01 & 1.11 \\
\hline Total soluble solids ( $\left.{ }^{\circ} b r i x\right)$ & $4.00^{* *}$ & 0.31 & 0.99 & 0.02 & 1.12 \\
\hline Beta-carotene & 0.011 & 0.003 & 0.0063 & 0.0006 & 0.27 \\
\hline Moisture content (\%) & $3.97 * *$ & 1.13 & 1.89 & 0.825 & 0.85 \\
\hline Dry matter content (\%) & 0.03 & 0.0047 & 0.014 & 0.0003 & 0.568 \\
\hline
\end{tabular}

Table.2 Estimates of gca values of parents for growth and yield traits of pumpkin

\begin{tabular}{|c|c|c|c|c|c|c|c|c|c|c|c|}
\hline Hybrids & $\begin{array}{c}\text { Days to } \\
\text { first } \\
\text { male } \\
\text { flower }\end{array}$ & $\begin{array}{c}\text { Days to } \\
\text { first } \\
\text { female } \\
\text { flower }\end{array}$ & $\begin{array}{c}\text { Nodes to } \\
\text { first } \\
\text { male } \\
\text { flower }\end{array}$ & $\begin{array}{l}\text { Node } \\
\text { to first } \\
\text { female } \\
\text { flower }\end{array}$ & Sex ratio & $\begin{array}{c}\text { Days to } \\
\text { first } \\
\text { harvest }\end{array}$ & $\begin{array}{c}\text { Number } \\
\text { of } \\
\text { fruits } \\
\text { per vine }\end{array}$ & $\begin{array}{c}\text { Average } \\
\text { fruit } \\
\text { weight }\end{array}$ & $\begin{array}{l}\text { Fruit } \\
\text { length }\end{array}$ & $\begin{array}{c}\text { Fruit } \\
\text { diameter }\end{array}$ & $\begin{array}{l}\text { Yield/ } \\
\text { plant }\end{array}$ \\
\hline $\mathbf{P}_{1}$ & -0.19 & $-1.11 * *$ & $-0.38 * *$ & 0.37 & -0.05 & -0.29 & $-0.21 * *$ & $2.49 * *$ & $4.38 * *$ & $4.14 * *$ & $1.73 * *$ \\
\hline $\mathbf{P}_{2}$ & $0.70 * *$ & -0.23 & -0.07 & -0.26 & -0.30 & $-1.64 * *$ & 0.05 & $-1.27 * *$ & $-2.54 * *$ & $-2.25 * *$ & $-1.39 * *$ \\
\hline $\mathbf{P}_{3}$ & $-0.39 *$ & 0.13 & $0.35^{*}$ & -0.31 & 0.15 & -0.17 & $0.16^{* *}$ & $-1.02 * *$ & $-3.53 * *$ & $-6.98 * *$ & $-0.97 * *$ \\
\hline $\mathbf{P}_{4}$ & $-0.63 * *$ & 0.29 & $-0.39 * *$ & -0.49 & $2.06 * *$ & $0.74 *$ & $-0.22 * *$ & 0.18 & $-0.37 * *$ & $0.85 * *$ & $1.74 * *$ \\
\hline $\mathbf{P}_{5}$ & $-0.58 * *$ & $0.44 * *$ & 0.06 & -0.15 & 0.082 & 0.036 & $0.24 * *$ & -0.02 & $2.25^{* *}$ & $3.50 * *$ & $0.39 * *$ \\
\hline $\mathbf{P}_{6}$ & $1.11 * *$ & $0.48 * *$ & $0.48 * *$ & $0.85^{*}$ & $-1.94 * *$ & $1.33 * *$ & 0.06 & -0.35 & 0.13 & $0.73 * *$ & $-0.71 * *$ \\
\hline SE & 0.19 & 0.15 & 0.13 & 0.30 & 0.15 & 0.28 & 0.02 & 0.25 & 0.13 & 0.26 & 0.03 \\
\hline CD (5\%) & 0.23 & 0.23 & 0.73 & 0.78 & 0.45 & 0.32 & 0.43 & 4.32 & 0.23 & 0.32 & 0.26 \\
\hline
\end{tabular}


Int.J.Curr.Microbiol.App.Sci (2018) 7(3): 3033-3039

Table.3 Estimates of $s c a$ values of hybrids for growth and yield traits of pumpkin

\begin{tabular}{|c|c|c|c|c|c|c|c|c|}
\hline Hybrids & $\begin{array}{l}\text { Number } \\
\text { of primary } \\
\text { branches }\end{array}$ & $\begin{array}{l}\text { Days } \\
\text { to first } \\
\text { male } \\
\text { flower }\end{array}$ & $\begin{array}{c}\text { Days to } \\
\text { first } \\
\text { female } \\
\text { flower }\end{array}$ & $\begin{array}{c}\text { Nodes to } \\
\text { first } \\
\text { male } \\
\text { flower }\end{array}$ & $\begin{array}{c}\text { Nodes to } \\
\text { first } \\
\text { female } \\
\text { flower }\end{array}$ & $\begin{array}{c}\text { Sex } \\
\text { Ratio }\end{array}$ & $\begin{array}{c}\text { Days to } \\
\text { first } \\
\text { harvest }\end{array}$ & $\begin{array}{l}\text { Number } \\
\text { of fruits } \\
\text { per vine }\end{array}$ \\
\hline $\mathbf{P}_{1} \times \mathbf{P}_{2}$ & $1.28 * *$ & 0.24 & $-1.27 * *$ & 0.05 & -1.07 & $-0.72 *$ & -0.26 & $0.15^{*}$ \\
\hline $\mathbf{P}_{1} \times \mathbf{P}_{3}$ & -0.78 & 0.72 & -0.61 & -0.65 & 1.34 & -0.04 & 0.37 & $0.16^{*}$ \\
\hline $\mathbf{P}_{1} \times \mathbf{P}_{4}$ & 0.52 & -0.63 & -0.25 & 0.38 & -0.39 & 0.14 & -0.77 & $-0.19^{* *}$ \\
\hline $\mathbf{P}_{1} \times \mathbf{P}_{5}$ & 0.20 & -0.07 & 0.71 & 0.16 & 1.07 & $1.97 * *$ & $2.25 * *$ & $-0.32 * *$ \\
\hline $\mathbf{P}_{1} \times \mathbf{P}_{6}$ & -0.64 & 0.39 & 0.53 & -0.53 & $-2.02 * *$ & 0.29 & $-1.65^{*}$ & -0.05 \\
\hline $\mathbf{P}_{2} \times \mathbf{P}_{1}$ & 0.00 & -0.79 & $-2.94 * *$ & -0.14 & -1.27 & -0.66 & $1.79 *$ & -0.13 \\
\hline $\mathbf{P}_{2} \times \mathbf{P}_{3}$ & 0.17 & -0.72 & 0.33 & $-0.96^{* *}$ & 0.31 & -0.04 & 0.39 & 0.03 \\
\hline $\mathbf{P}_{2} \times \mathbf{P}_{4}$ & 0.14 & 0.26 & $-0.74^{*}$ & -0.43 & -0.23 & 0.24 & 1.08 & -0.12 \\
\hline $\mathbf{P}_{2} \times \mathbf{P}_{5}$ & $-1.25^{*}$ & -0.04 & $0.88^{*}$ & 0.15 & -0.95 & 0.57 & 0.24 & 0.02 \\
\hline $\mathbf{P}_{2} \times \mathbf{P}_{6}$ & 0.81 & 0.68 & -0.16 & $0.81^{*}$ & $1.89 * *$ & 0.06 & -0.59 & $-0.16^{*}$ \\
\hline $\mathbf{P}_{3} \times \mathbf{P}_{1}$ & 0.0 & 0.14 & $-2.75^{* *}$ & 0.08 & -1.25 & $-1.11 * *$ & 0.62 & -0.11 \\
\hline $\mathbf{P}_{3} \times \mathbf{P}_{2}$ & 0.08 & -0.08 & -0.58 & 0.21 & $2.14^{*}$ & 0.24 & -0.53 & 0.01 \\
\hline $\mathbf{P}_{3} \times \mathbf{P}_{4}$ & -0.91 & 0.52 & -0.02 & -0.04 & -1.37 & -0.26 & -0.78 & $-0.17 * *$ \\
\hline $\mathbf{P}_{3} \times \mathbf{P}_{5}$ & $1.34 * *$ & 0.38 & -0.42 & -0.33 & 0.37 & 0.31 & -0.07 & $0.16^{*}$ \\
\hline $\mathbf{P}_{3} \times \mathbf{P}_{6}$ & -0.17 & -0.53 & -0.16 & $0.97 * *$ & 0.07 & -0.23 & 0.41 & -0.08 \\
\hline $\mathbf{P}_{4} \times \mathbf{P}_{1}$ & -0.08 & 1.03 & 0.32 & -0.62 & 0.66 & $-3.14^{* *}$ & 0.53 & $-0.34 * *$ \\
\hline $\mathbf{P}_{4} \times \mathbf{P}_{2}$ & -0.16 & 0.33 & 0.82 & 0.63 & 1.58 & $-2.72 * *$ & $-2.63^{* *}$ & 0.07 \\
\hline $\mathbf{P}_{4} \times \mathbf{P}_{3}$ & 0.00 & 0.54 & $1.75^{* *}$ & 0.03 & 0.33 & $-1.44^{* *}$ & -0.29 & -0.05 \\
\hline $\mathbf{P}_{4} \times \mathbf{P}_{5}$ & $-1.09 *$ & 0.13 & -0.38 & -0.49 & 0.62 & $-0.75^{*}$ & -0.87 & $0.27 * *$ \\
\hline $\mathbf{P}_{4} \times \mathbf{P}_{6}$ & -0.28 & -0.72 & -0.49 & $-0.78 *$ & $1.58 *$ & $1.27 * *$ & 0.25 & $0.15^{*}$ \\
\hline $\mathbf{P}_{5} \times \mathbf{P}_{1}$ & 0.0 & -0.81 & $-1.83^{* *}$ & 0.56 & $-1.86^{*}$ & $-2.91 * *$ & $-2.48^{* *}$ & 0.11 \\
\hline $\mathbf{P}_{5} \times \mathbf{P}_{2}$ & 0.00 & $1.13^{*}$ & $1.05^{*}$ & $-1.08^{* *}$ & 0.36 & -0.68 & $-2.96^{* *}$ & 0.01 \\
\hline $\mathbf{P}_{5} \times \mathbf{P}_{3}$ & 0.00 & $-1.13^{*}$ & $-1.04 *$ & -0.31 & -1.58 & -0.29 & -0.11 & $-0.33 * *$ \\
\hline $\mathbf{P}_{5} \times \mathbf{P}_{4}$ & 0.00 & -0.92 & $-1.63^{* *}$ & 0.61 & -0.16 & $2.48 * *$ & 1.07 & 0.05 \\
\hline $\mathbf{P}_{5} \times \mathbf{P}_{6}$ & 0.97 & -0.24 & $0.94 *$ & 0.42 & $-1.41 *$ & $-0.83 *$ & -0.15 & -0.07 \\
\hline$P_{6} \times P_{1}$ & 0.00 & 0.64 & $1.24 * *$ & -0.52 & 0.83 & $0.89 *$ & 0.63 & -0.15 \\
\hline $\mathbf{P}_{6} \times P_{2}$ & 0.41 & -0.66 & 0.39 & $0.83^{*}$ & -1.33 & $1.74 * *$ & $-2.68 * *$ & $-0.19^{*}$ \\
\hline $\mathbf{P}_{6} \times \mathbf{P}_{3}$ & -0.66 & -1.18 & -0.69 & 0.27 & -0.41 & $1.92 * *$ & -1.51 & 0.03 \\
\hline $\mathbf{P}_{6} \times \mathbf{P}_{4}$ & 0.50 & -0.25 & $1.35^{* *}$ & $-0.75^{*}$ & -0.91 & $3.37 * *$ & $-1.94 *$ & -0.16 \\
\hline $\mathbf{P}_{6} \times \mathbf{P}_{5}$ & -0.66 & -0.94 & -0.05 & -0.41 & 0.75 & 0.35 & $-2.67 * *$ & $0.23 * *$ \\
\hline $\mathrm{CD}(5 \%)$ & 0.45 & 0.18 & 0.59 & 0.72 & 0.26 & 0.34 & 0.32 & 0.23 \\
\hline CD (1\%) & 0.58 & 0.24 & 0.62 & 0.86 & 0.56 & 0.46 & 0.52 & 0.46 \\
\hline
\end{tabular}


Table.4 Estimates of $s c a$ values of hybrids for growth and yield traits of pumpkin

\begin{tabular}{|c|c|c|c|c|c|c|c|c|c|}
\hline Hybrids & $\begin{array}{c}\text { Average } \\
\text { fruit } \\
\text { weight } \\
\text { (Kg) }\end{array}$ & $\begin{array}{c}\text { Fruit } \\
\text { length } \\
(\mathrm{cm})\end{array}$ & $\begin{array}{c}\text { Fruit } \\
\text { diameter } \\
(\mathbf{c m})\end{array}$ & $\begin{array}{c}\text { Flesh } \\
\text { thickness } \\
(\mathrm{cm})\end{array}$ & $\begin{array}{c}\text { Number } \\
\text { of seeds } \\
\text { per } \\
\text { fruit }\end{array}$ & $\begin{array}{c}\text { Yield } \\
\text { per } \\
\text { plant } \\
(\mathrm{Kg})\end{array}$ & $\begin{array}{c}\text { Total } \\
\text { Soluble } \\
\text { solids } \\
\text { (TSS) } \\
\end{array}$ & $\begin{array}{c}\text { Beta } \\
\text { Carotene }\end{array}$ & $\begin{array}{c}\text { Dry } \\
\text { matter } \\
\text { content }\end{array}$ \\
\hline $\mathbf{P}_{1} \times \mathbf{P}_{2}$ & 0.16 & $-1.51 * *$ & -0.63 & 0.05 & 2.83 & $-0.44 * *$ & -0.09 & $-0.03 *$ & $0.08 * *$ \\
\hline $\mathbf{P}_{1} \times \mathbf{P}_{3}$ & 0.23 & -0.55 & -0.36 & $0.17^{*}$ & 2.78 & $0.35 * *$ & $-0.44 * *$ & -0.02 & $-0.02 *$ \\
\hline $\mathbf{P}_{1} \times \mathbf{P}_{4}$ & 0.07 & -0.13 & 0.07 & 0.08 & $-7.50 *$ & $-1.02 * *$ & $0.29 * *$ & -0.02 & $0.02 *$ \\
\hline $\mathbf{P}_{1} \times \mathbf{P}_{5}$ & 1.08 & $3.62 * *$ & 0.82 & $0.14^{*}$ & $15.93 * *$ & $2.92 * *$ & $-0.68 * *$ & -0.01 & $0.04 * *$ \\
\hline $\mathbf{P}_{1} \times \mathbf{P}_{6}$ & $-1.39 *$ & $1.31 * *$ & $1.86^{* *}$ & $-0.34^{* *}$ & $-7.55^{*}$ & $-0.76^{* *}$ & $-0.21^{*}$ & -0.06 & $-0.04 * *$ \\
\hline $\mathbf{P}_{2} \times \mathbf{P}_{1}$ & $3.93 * *$ & $5.89 * *$ & $5.62 * *$ & $0.24 * *$ & $40.01 * *$ & $3.05 * *$ & $-0.36^{* *}$ & $-0.06^{* *}$ & -0.05 \\
\hline $\mathbf{P}_{2} \times \mathbf{P}_{3}$ & $-0.19 *$ & 0.37 & $1.86^{* *}$ & -0.19 & 4.42 & $0.41 * *$ & 0.03 & $0.08 * *$ & -0.09 \\
\hline $\mathbf{P}_{2} \times \mathbf{P}_{4}$ & -0.43 & 0.28 & 0.67 & 0.01 & $19.29 * *$ & $0.72 * *$ & -0.14 & -0.08 & 0.01 \\
\hline $\mathbf{P}_{2} \times \mathbf{P}_{5}$ & -0.16 & -0.54 & $1.64 * *$ & -0.08 & 2.96 & $-0.64 * *$ & $-0.31 * *$ & $-0.03 *$ & 0.01 \\
\hline $\mathbf{P}_{2} \times \mathbf{P}_{6}$ & 0.23 & $1.97 * *$ & -1.15 & $0.16^{*}$ & -4.10 & 0.04 & $-0.22 *$ & 0.02 & $0.03 * *$ \\
\hline $\mathbf{P}_{3} \times \mathbf{P}_{1}$ & $2.48 * *$ & $5.86^{* *}$ & $10.85^{* *}$ & -0.09 & $7.86^{*}$ & $2.32 * *$ & $-0.86 * *$ & $-0.16^{* *}$ & $0.08 * *$ \\
\hline $\mathbf{P}_{3} \times \mathbf{P}_{2}$ & -0.23 & 0.18 & $4.67 * *$ & 0.09 & $-24.76^{* *}$ & -0.35 & $-0.96 * *$ & -0.02 & 0.01 \\
\hline $\mathbf{P}_{3} \times \mathbf{P}_{4}$ & -0.49 & 0.16 & -0.16 & 0.07 & -3.02 & -0.05 & -0.03 & 0.02 & $0.05 * *$ \\
\hline $\mathbf{P}_{3} \times \mathbf{P}_{5}$ & -0.09 & -0.46 & 0.38 & -0.05 & $-8.15^{*}$ & $-0.50 * *$ & 0.16 & 0.02 & $-0.03 * *$ \\
\hline $\mathbf{P}_{3} \times \mathbf{P}_{6}$ & 0.64 & 0.32 & 0.68 & 0.05 & $7.67 *$ & $0.32 * *$ & 0.05 & -0.02 & 0.09 \\
\hline $\mathbf{P}_{4} \times \mathbf{P}_{1}$ & 0.73 & $3.41 * *$ & $2.65 * *$ & $-0.18^{*}$ & -7.83 & -1.55 & $-1.09 *$ & $0.07 * *$ & $0.08 * *$ \\
\hline $\mathbf{P}_{4} \times \mathbf{P}_{2}$ & -0.77 & $-2.85^{* *}$ & $-4.35 * *$ & -0.07 & $-58.58 * *$ & $-3.33 * *$ & $-0.94 * *$ & -0.03 & $0.11 * *$ \\
\hline $\mathbf{P}_{4} \times \mathbf{P}_{3}$ & $-1.01^{* *}$ & $-2.7^{* *}$ & $-8.31 * *$ & -0.06 & -5.83 & $-3.16^{* *}$ & 0.07 & 0.08 & $0.08 * *$ \\
\hline $\mathbf{P}_{4} \times \mathbf{P}_{5}$ & 0.07 & 0.13 & 0.36 & $-0.23 * *$ & 4.66 & -0.39 & 0.08 & $-0.02 * *$ & -0.07 \\
\hline $\mathbf{P}_{4} \times \mathbf{P}_{6}$ & 0.21 & -0.38 & -0.88 & $0.15^{*}$ & $2.55^{* *}$ & $0.22 * *$ & $-0.25 *$ & 0.02 & -0.02 \\
\hline $\mathbf{P}_{5} \times \mathbf{P}_{1}$ & -0.07 & $-2.25^{* *}$ & $-1.63^{*}$ & $-0.48 * *$ & $-36.09 * *$ & $0.97 * *$ & $-0.47 * *$ & $0.06 * *$ & $0.07 * *$ \\
\hline $\mathbf{P}_{5} \times \mathbf{P}_{2}$ & -0.63 & $-4.58 * *$ & $-1.63 *$ & $-0.19 *$ & $33.88^{* *}$ & $0.61 * *$ & $-0.94 * *$ & 0.01 & $0.05 * *$ \\
\hline $\mathbf{P}_{5} \times \mathbf{P}_{3}$ & -0.13 & $-4.76^{* *}$ & $-6.87 * *$ & $-0.23 * *$ & 7.04 & 0.16 & $-0.58 * *$ & -0.05 & $0.18 * *$ \\
\hline $\mathbf{P}_{5} \times \mathbf{P}_{4}$ & 1.11 & $-1.71 * *$ & $-10.27 * *$ & $0.28^{*}$ & -3.29 & $2.92 * *$ & -0.13 & $0.07 * *$ & $0.07 * *$ \\
\hline $\mathbf{P}_{5} \times \mathbf{P}_{6}$ & -0.42 & $-1.84 * *$ & 0.07 & -0.06 & -4.97 & $-0.24 * *$ & $0.57 * *$ & -0.09 & -0.01 \\
\hline $\mathbf{P}_{6} \times \mathbf{P}_{1}$ & 1.29 & $1.77 * *$ & $1.77^{*}$ & -0.08 & $7.65^{*}$ & $2.71 * *$ & $-0.45^{* *}$ & $0.09 * *$ & 0.05 \\
\hline $\mathbf{P}_{6} \times \mathbf{P}_{2}$ & -0.43 & $-4.61 * *$ & $-2.79 * *$ & -0.18 & $-57.88 * *$ & $-1.06^{* *}$ & $0.58 * *$ & -0.05 & $0.17 * *$ \\
\hline $\mathbf{P}_{6} \times \mathbf{P}_{3}$ & -0.92 & $-2.77 * *$ & $-8.68 * *$ & $-0.58 * *$ & $-19.19 * *$ & $-1.06^{* *}$ & $0.35 * *$ & 0.02 & $0.04 * *$ \\
\hline $\mathbf{P}_{6} \times \mathbf{P}_{4}$ & 1.23 & $1.50 * *$ & $2.22 * *$ & $-0.43 * *$ & -12.04 & $2.46 * *$ & $0.93 * *$ & $-0.05^{* *}$ & $0.09 * *$ \\
\hline $\mathbf{P}_{6} \times \mathbf{P}_{5}$ & -0.19 & $2.57 * *$ & $2.15^{* *}$ & $-0.36 * *$ & $-25.11 * *$ & $-0.98 * *$ & $1.24 * *$ & $-0.07 * *$ & $0.06 * *$ \\
\hline $\mathrm{CD}(5 \%)$ & 0.23 & 0.45 & 0.28 & 0.34 & 0.36 & 0.32 & 0.49 & 0.05 & 0.003 \\
\hline CD (1\%) & 0.43 & 0.64 & 0.32 & 0.48 & 0.54 & 0.48 & 0.52 & 0.07 & 0.009 \\
\hline
\end{tabular}


The ratio of $\sigma^{2} g / \sigma^{2} s$ was lesser than one for the character nodes to first female flower there by indicating preponderance of nonadditive (dominance) variation in expression of this trait.

Estimates of general combining ability effects (Table 2) showed that the parent $P_{1}$ is best combiner for days to first female flower (1.11), fruit weight (2.41), fruit length (4.38), fruit diameter (4.14) and yield per plant (1.73). $\mathrm{P}_{2}$ is best combiner for small sized fruits (0.21) which exhibited negative $g c a$ effects. The $\mathrm{P}_{3}$ for more number of fruits per plant (0.16), $\mathrm{P}_{4}$ for narrow sex ratio (2.06). Different set of parents with these types of results were reported by Lawande and Patil (1991).

The specific combining ability effects of $F_{1}$ are given in Table 3. It was observed that out 30 cross combinations four for number of primary branches, one for days to first male flower, seven for days to first female flower, four for nodes to first male flower, three for node to first female flower, seven for sex ratio, seven for days to first harvest, 13 for number of fruits per vine, five for average fruit weight, 19 for fruit length, 18 for fruit diameter, 15 for flesh thickness, 18 for number of seeds per fruit, 11 for yield per plant, six for total soluble solids, 16 for day matter contents exhibited significant sca effects in desirable direction, indicating presence of non-exploitation of hybrid vigour in these characters. Similar results of specific combining ability effects were also reported by Sundaram (2006), and Naliyadhara et al., (2010) in sponge gourd.

The earliness is measured in terms of days to first female flowering and first female flower node in pumpkin. This indicated by negatively significant values of specific combining ability. The crosses $\mathrm{P}_{3} \times \mathrm{P}_{1}(-2.04)$, $\mathrm{P}_{2} \times \mathrm{P}_{1}(-2.75), \mathrm{P}_{5} \times \mathrm{P}_{1}(-1.83)$ and $\mathrm{P}_{5} \times \mathrm{P}_{3}$ recorded negative significant values for days to first female flowering. The first female flowering node was significant negative in $\mathrm{P}_{1}$ $x \mathrm{P}_{6}(-2.04), \mathrm{P}_{5} \times \mathrm{P}_{6}(1.41)$ and $\mathrm{P}_{5} \times \mathrm{P}_{1}(-1.86)$ crosses. The sex ratio indicates number of male and female flowers per plant. The negative significant sca effects are favourable for getting more number of fruits and it was found in $\mathrm{P}_{4} \times \mathrm{P}_{1}(-3.14), \mathrm{P}_{5} \times \mathrm{P}_{1}(-2.91), \mathrm{P}_{4} \times$ $\mathrm{P}_{2}$ (-2.72), $\mathrm{P}_{3} \times \mathrm{P}_{1}(-1.11)$ and $\mathrm{P}_{1} \times \mathrm{P}_{2}(-0.72)$. Similar results were reported by Thangamani et al., (2011) in bitter gourd.

\section{Yield parameters}

Yield is the most important character in any crop breeding programme. The specific combining ability for small fruited type having less length and diameter is negatively significant is desirable effect. It was observed is $\mathrm{P}_{1} \times \mathrm{P}_{6}(-1.39,-1.31,-1.86), \mathrm{P}_{4} \times \mathrm{P}_{3}(-1.01$, $-2.70,-8.31)$ crosses. The significant positive sca effect observed for bigger sized fruit with more fruit length and diameter are $\mathrm{P}_{2} \times \mathrm{P}_{1}$ (3.93, 5.89, 5.62), $\mathrm{P}_{3} \times \mathrm{P}_{1}(2.48,5.86,10.85)$. These results are in conformity with findings of Sapovadiya et al., (2014) is water melon.

The higher sca effect on flesh thickness and number of seed per fruit was observed in $\mathrm{P}_{2} \mathrm{X}$ $P_{1}(0.24,40.01)$. The yield per plant was found in sca effect of $\mathrm{P}_{2} \times \mathrm{P}_{1}$ (3.05) and $\mathrm{P} 5 \mathrm{x}$ $\mathrm{P} 4$ (2.92). The significant positive effect sca for total soluble solids recorded in $\mathrm{P}_{6} \times \mathrm{P}_{5}$ (1.24), for beta carotene content in $\mathrm{P}_{6} \times \mathrm{P}_{1}$ (0.09) and for dry matter content in $\mathrm{P}_{5} \times \mathrm{P}_{3}$ (0.18) and $\mathrm{P}_{6} \times \mathrm{P}_{2}$ (0.17). These cross combinations with desirable $s c a$ could be well utilized in heterosis breeding as reported by Tewari et al., (2001) and Laxuman et al., (2012) (Table 4).

In the future breeding programme, the desirable general combiners viz. $\mathrm{P}_{1}, \mathrm{P}_{2}$ and $\mathrm{P}_{3}$ may be utilized for crop improvement studies of pumpkin. The cross combinations for small 
fruited type $\mathrm{P}_{1} \times \mathrm{P}_{6}$ and $\mathrm{P}_{4} \times \mathrm{P}_{3}$ and for big fruited type $\mathrm{P}_{2} \times \mathrm{P}_{1}$ and $\mathrm{P}_{3} \times \mathrm{P}_{1}$ may be tested different locations for assessing yield stability.

\section{References}

Devadas, V.S. 1993. Genetic studies on fruit and seed yield and quality in bitter gourd (Momordica charantia L.). Ph.D. Thesis, Department of Olericulture, Faculty of Horticulture, Tamil Nadu Agricultural University, Coimbatore.

Gopalakrishnan, R. 1986. Oiallel analysis in bitter gourd (Momordica charantia L.). M.Sc. (Hort) Thesis, TNAU, Coimbatore.

Gopalakrishnan, T.R., Gopalakrishnan, P.K. and K.V. Peter. 1980. Variability, heritability and correlation among some polygenic characters in pumpkin. Indian J. Agric. Sci., 50 (12): 925-930.

Griffing, J.B. 1956. Concept of general and specific combining ability in relation to diallel crossing systems. Aust. J. Biol. Sci., 9: 463-493.

Lawande, K.E. and Patil, A.V. 1991. Studies on gene action in bitter gourd (Momordica charantia L.).Veg. Sci., 18(2): 192-199.

Laxuman, Patil, Salimath, S.A., Dharmatti, P.M., Byadgi, P.R., and Nirmala Yenagi, A.S. 2012. Heterosis and combining ability analysis for productivity traits in bitter gourd (Momordica charcintia L.) Karnataka Journal of Agric. Sci 25(1):9-13.
Naliyadhara, M.V., Dhaduk, L.K., Barad, A.V. and Mehta, D.R. 2010. Combining ability analysis in sponge gourd (Luffa cylindrica (Roem.) L.) Veg. Sci. 37(1):21-24.

Sapovadiya, M.H., Mehta, D.R., Dhaduk, H.L. and Babariya, C.A. 2014. Combining ability in Water melon (Citrullus lanatus (Thunb) (Mansf.). Electronic Journal of plant breeding, 5(3): 327-330.

Sprague, G.F. and Tatum, L.A. 1942. General verses specific combining ability in single cross corn. J. Amer. Soc. Agron., 34: 923-932.

Sundaram, V. 2006. Studies on genetics of yield and yield components in bitter gourd (Momordica charantia L.) under salinity. Ph.D. (Hort) thesis, TNAU, Coimbatore.

Tewari, D. Ram, H.H. and Jaiswal, H.R. 2001. Studies on heterosis and combining ability in indigenous bitter gourd (Momordica charantia L.) for fruit yield. Veg. sci. 28: 106-108.

Thangamani, C. and Pugalendhi, L. 2013. Combining ability studies is bitter gourd (Momordica charantia L.)

Thangamani, C., Pugalendhi, L., Sumathi, T, Kavitha, C., and Rajashree, V. 2011. Estimation of combining ability and heterosis for yield and quality characters in bitter gourd (Momordica charantia L), Electronic Journal of Plant Breeding, 2(1):62-66.

\section{How to cite this article:}

Marxmathi, P., V. Krishnamoorthy and Thankaraj, P. 2018. Combining Ability Studies in Pumpkin (Cucurbita moschata Duch ex Poir). Int.J.Curr.Microbiol.App.Sci. 7(03): 3033-3039. doi: https://doi.org/10.20546/ijcmas.2018.703.352 